2006-9

\title{
Maximizing Miniature Aerial Vehicles
}

Stephen Griffiths

Scientific Systems Company, Inc.

Jeffery Brian Saunders

Brigham Young University - Provo, saunders.jeff@gmail.com

Andrew Curtis

Brigham Young University - Provo

Blake Barber

Brigham Young University - Provo

Timothy W. McLain

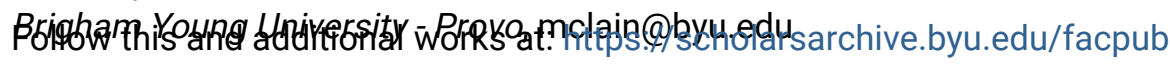

Part of the Electrical and Computer Engineering Commons, and the Mechanical Engineering Seenextipage for additional authors

\section{Original Publication Citation}

Griffiths, S., Saunders, J., Curtis, A., Barber, B., McLain, T., and Beard, R. Maximizing Miniature Aerial Vehicles, IEEE Robotics and Automation, Special Issue on Unmanned Aerial Vehicles: Enabling Technologies \& Roadmap for Autonomy, vol. 13, no. 3, pp. 34-43, September 2006. Invited submission.

\section{BYU ScholarsArchive Citation}

Griffiths, Stephen; Saunders, Jeffery Brian; Curtis, Andrew; Barber, Blake; McLain, Timothy W.; and Beard, Randal W., "Maximizing Miniature Aerial Vehicles" (2006). Faculty Publications. 1530.

https://scholarsarchive.byu.edu/facpub/1530

This Peer-Reviewed Article is brought to you for free and open access by BYU ScholarsArchive. It has been accepted for inclusion in Faculty Publications by an authorized administrator of BYU ScholarsArchive. For more information, please contact ellen_amatangelo@byu.edu. 
Authors

Stephen Griffiths, Jeffery Brian Saunders, Andrew Curtis, Blake Barber, Timothy W. McLain, and Randal W. Beard 


\title{
Obstacle and Terrain Avoidance for Miniature Aerial Vehicles
}

\author{
Stephen Griffiths, Jeff Saunders, Andrew Curtis, Blake Barber, \\ Tim McLain, Senior Member, IEEE, and Randy Beard Senior Member, IEEE
}

\section{INTRODUCTION}

Unmanned aerial vehicles (UAVs) are playing increasingly prominent roles in defense programs and strategy around the world. Technology advancements have enabled the development of large UAVs (e.g., Global Hawk, Predator) and the creation of smaller, increasingly capable UAVs. The focus of this article is on smaller fixed-wing miniature aerial vehicles (MAVs), which range in size from $1 / 4$ to $2 \mathrm{~m}$ in wingspan. As recent conflicts have demonstrated, there are numerous military applications for MAVs including reconnaissance, surveillance, battle damage assessment, and communications relays.

Civil and commercial applications are not as well developed, although potential applications are extremely broad in scope. Possible applications for MAV technology include environmental monitoring (e.g., pollution, weather, and scientific applications), forest fire monitoring, homeland security, border patrol, drug interdiction, aerial surveillance and mapping, traffic monitoring, precision agriculture, disaster relief, adhoc communications networks, and rural search and rescue. For many of these applications to develop to maturity, the reliability of MAVs will need to increase, their capabilities will need to be extended further, their ease of use will need to be improved, and their cost will have to come down. In addition to these technical and economic challenges, the regulatory challenge of integrating UAVs into the national and international air space needs to be overcome.

Critical to the more widespread use of MAVs is making them easy to use by non-pilots, such as scientists, forest fire fighters, law enforcement officers, or military ground troops. One key capability for facilitating ease of use is the ability to sense and avoid obstacles, both natural and man made. Many of the applications cited require MAVs to fly at low altitudes in close proximity to structures or terrain. For example, the ability to fly through city canyons and around high-rise buildings is envisioned for future homeland security operations. For MAVs to be effective tools, the challenge of operating in complex environments must be automated, allowing the operator to concentrate on the task at hand.

Performing obstacle and terrain avoidance from a fixedwing MAV platform is challenging for several reasons. The limited payload and power availability of MAV platforms places significant restrictions on the size, weight, and power requirements of potential sensors. Sensors such as scanning

The authors are with Brigham Young University, Provo, Utah 84602. T. McLain (mclain@byu.edu) is the corresponding author.
LADAR and RADAR are typically too large and heavy for MAVs. Related to limits on sensor payload are those on computing resources. For most MAVs, the primary computational resource is the excess capacity in the autopilot microcontroller. Additional computational capacity can be added, but computers such as PC104-based systems generally exceed the payload capacity of MAVs: smaller microcontrollers are typically used.

Another challenge posed by fixed-wing MAVs is that they move fast: ground speeds are often in the range of 10 to $20 \mathrm{~m} / \mathrm{s}$ (22 to $44 \mathrm{mph}$ ). Contrary to the computational limits imposed, obstacle avoidance algorithms must execute and act quickly. Unlike ground robots and unmanned rotorcraft, fixed-wing MAVs cannot stop or slow down while avoidance algorithms process sensor information or plan maneuvers. Reactions must be immediate. Obstacle sensing is further complicated by the fact that sensor readings are altered by changes in aircraft attitude, especially the rolling motions that occur during turns. Attitude changes affect not only the pointing direction of the sensor, but also cause motion of fixed objects in the field of view. Obstacle and terrain detection must account for the effects of aircraft attitude changes for avoidance maneuvers to be successful. All of the challenges associated with MAV obstacle and terrain avoidance are compounded by the reality that for MAVs, mistakes are costly or even catastrophic, as crashes can result in damage to or loss of the MAV and failure to complete the objectives of the flight.

As evidenced by the recent DARPA Grand Challenge, capable obstacle avoidance and terrain navigation systems have been developed for ground vehicles. Obstacle avoidance and path planning have been active areas of research for many years and the associated robotics literature is immense. While providing a guiding influence, most of the proposed methods fail to deal with the sensing and computational challenges imposed by the limited payload capabilities of MAVs.

As autonomous MAVs and feasible obstacle sensors are recent technological developments, the body of experimental research directed specifically toward MAV obstacle and terrain avoidance is small. Related to terrain avoidance is work focused on utilizing vision processing techniques to estimate height above ground. Chahl, et al. demonstrated that mimicking the landing behavior of bees, by maintaining constant optic flow during a landing maneuver, could be used to successfully control the descent of a MAV [1]. Development of lightweight sensors for measurement of optic flow has enabled their use in MAVs [2], [3], [4]. Barrows, et al. have demonstrated that these sensors can be used to follow undulations in terrain with low-flying MAVs [5]. 
This article presents MAV obstacle and terrain avoidance research performed at Brigham Young University (BYU). Our work builds on the notion of utilizing useful but imperfect map information to plan nominal paths through city or mountain terrain. Because maps may be limited in resolution, out of date, or offset in location, MAVs must also utilize sensory information to detect and avoid obstacles unknown to the path planner. In this article, we present research utilizing laser range finder and optic flow sensors to detect obstacles and terrain. Avoidance algorithms using this sensor information are discussed briefly and flight test results from our MAVs are presented.

\section{B YU Miniature Aerial Vehicle Platforms}

Over the past five years, BYU has been involved in the development of MAV airframes, autopilots, user interfaces, sensors, and control algorithms. This section describes the experimental platform developed specifically for the obstacle avoidance research described in this article.

\section{A. Airframe}

Figure 1 shows the airframe used for obstacle avoidance experiments. The airframe has a $1.5 \mathrm{~m}$ wingspan and was constructed with an EPP foam core covered with Kevlar. This design was selected for its durability, useable payload, ease of component installation, and flight characteristics. The airframe can carry a $0.4 \mathrm{~kg}$ payload and can remain in flight for over 45 minutes at a time. The collision avoidance sensors that are embedded in the airframe include three optic-flow sensors, one laser ranger, and two electro-optical cameras as shown in Figure 2. Additional payload includes the Kestrel autopilot, batteries, a $1000 \mathrm{~mW}, 900 \mathrm{MHz}$ radio modem, a 12-channel GPS receiver, and a video transmitter.

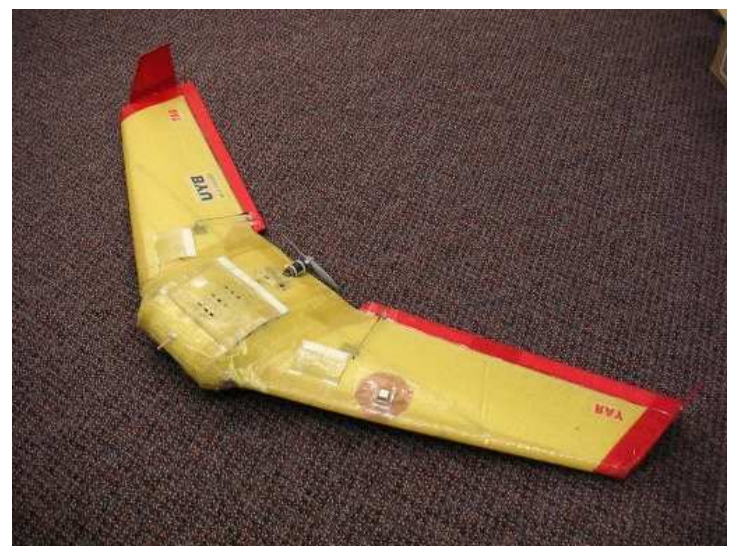

Fig. 1. Airframe used for collision avoidance experiments.

\section{B. Kestrel Autopilot}

The collision avoidance algorithms described in this paper were implemented on Procerus Technologies' Kestrel Autopilot version 2.2 [6]. The autopilot is equipped with a Rabbit $340029 \mathrm{MHz}$ processor, three-axis rate gyros, three-axis accelerometers, absolute and differential pressure sensors, and

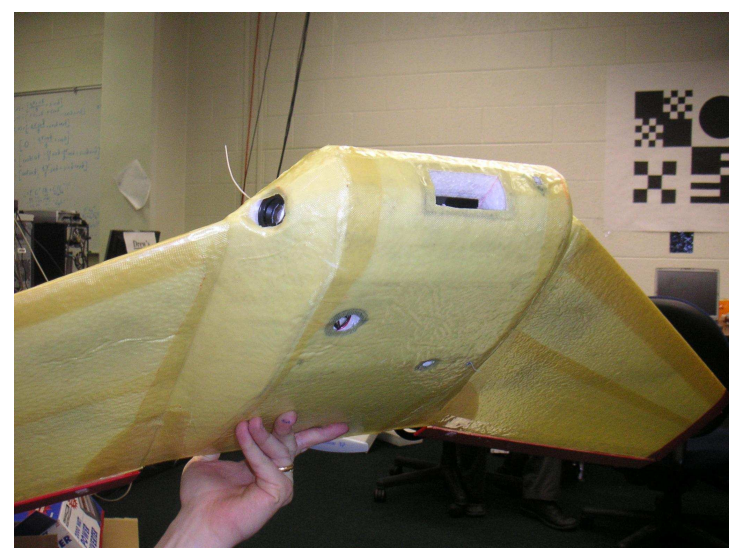

Fig. 2. Sensors used for collision avoidance. The round hole on the right and the large hole on the belly are the optic flow sensors. The square hole in the center is the laser ranger, and the other two round holes are for electro-optical cameras.

a variety of interface ports. The autopilot measures $3.8 \times 5.1 \times$ $1.9 \mathrm{~cm}$ and weighs 18 grams. The autopilot also serves as a data acquisition device and is able to log 175 kbytes of user-selectable telemetry at rates up to $60 \mathrm{~Hz}$. The optic flow sensors and the laser ranger used in this paper are connected directly to the autopilot and the collision avoidance algorithms are executed on-board the Rabbit processor.

\section{Optic Flow Sensors}

The MAV is equipped with three optic-flow sensors. Two of the optic-flow sensors are forward looking but swept back from the nose by $\alpha=60$ degrees. The third optic flow sensor points down to determine the height above ground. The opticflow sensors, shown in Figure 3, are constructed by attaching a lens to an Agilent ADNS-2610 optical mouse sensor. The ADNS-2610 has a small form factor, measuring only $10 \mathrm{~mm}$ by $12.5 \mathrm{~mm}$ and runs at 1500 frames per second. It requires a light intensity of at least $80 \mathrm{~mW} / \mathrm{m}^{2}$ at a wavelength of $639 \mathrm{~nm}$ or $100 \mathrm{~mW} / \mathrm{m}^{2}$ at a wavelength of $875 \mathrm{~nm}$. The ADNS-2610 measures the flow of features across an 18 by 18 pixel CMOS imager. It outputs two values, $\delta p_{x}$ and $\delta p_{y}$, representing the total optic flow across the sensor's field of view in both the $x$ and $y$ directions. The flow data in the camera $y$ direction corresponds to lateral motion of the MAV and is ignored.

Figure 4 indicates how distance is computed using the optic flow sensor. The optical mouse chip outputs an optic flow displacement $\left(\delta p_{x}, \delta p_{y}\right)^{T}$ at its internal sample rate $(1500 \mathrm{~Hz})$. Since the collision avoidance loop is executed at $T_{s}=20 \mathrm{~Hz}$, the total optical displacement is integrated over $T_{s}$ to produce $\left(\Delta p_{x}, \Delta p_{y}\right)$. The distance to the object $D$ is related to the measured distance $d$ by the expression

$$
D=d \cos \phi \sin \alpha,
$$

where $\phi$ is the roll angle of the MAV. From geometry, the measured distance to the object is given by

$$
d=\frac{V_{\mathrm{gps}} T_{s}}{\tan \left(\frac{\lambda_{\mathrm{eff}}}{2}\right)},
$$




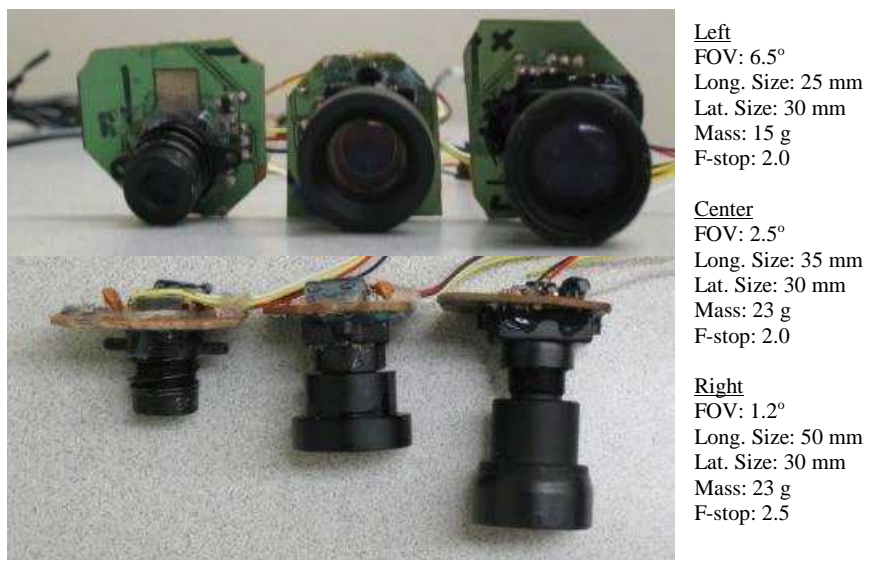

Fig. 3. Optic flow sensors with three different lens configurations: 1.2, 2.5 , and 6.5 degree field-of-view. The optic flow sensors are constructed by attaching a lens to an optical mouse chip.

where $\lambda_{\text {eff }}$ is the effective field-of-view. The effective field of view is given by

$$
\lambda_{\text {eff }}=\lambda_{\text {cam }} \frac{\Delta p_{x}}{P_{x}}-\dot{\chi} T_{s},
$$

where $\lambda_{\text {cam }}$ is the field of view of the camera, $P_{x}$ is the size of the pixel array along the direction of motion, and $\dot{\chi}$ is the yaw rate with respect to the ground. Using similar reasoning for left-looking and down-looking optic flow sensors we can derive the following expression:

$$
\begin{aligned}
& D_{\text {right }}=\frac{V_{\mathrm{gps}} T_{s}}{\tan \left(\frac{\lambda_{\mathrm{cam}} D_{\text {right }} p_{x}}{2 P_{x}}-\frac{\dot{\chi} T_{s}}{2}\right)} \cos \phi \sin \alpha \\
& D_{\text {left }}=\frac{V_{\mathrm{gps}} T_{s}}{\tan \left(\frac{\lambda_{\text {cam }} D_{\text {left }} p_{x}}{2 P_{x}}+\frac{\dot{\chi} T_{s}}{2}\right)} \cos \phi \sin \alpha \\
& D_{\text {down }}=\frac{V_{\mathrm{gps}} T_{s}}{\tan \left(\frac{\lambda_{\text {cam }} D_{\text {down }} p_{x}}{2 P_{x}}-\frac{\dot{\theta} T_{s}}{2}\right)} \cos \theta \cos \phi .
\end{aligned}
$$

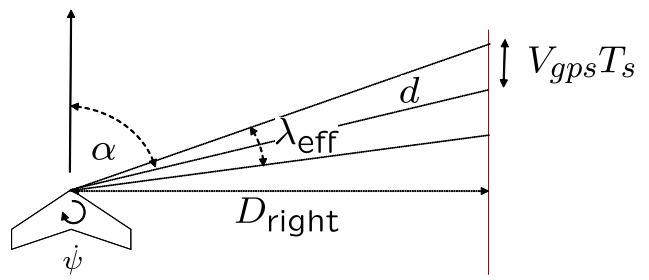

Fig. 4. The optic flow sensor is used to compute the distance to an obstacle based on the distance traveled between samples $\left(V_{\mathrm{gps}} T_{s}\right)$ and the effective field of view $\lambda$.

\section{Laser Ranger}

For the experiments discussed in this paper we used the Opti-Logic RS400 Laser rangefinder. The range finder has a range of $400 \mathrm{~m}$ with an update rate of $3 \mathrm{~Hz}$. It weights 170 grams and consumes $1.8 \mathrm{~W}$ of power. Figure 2 shows the laser ranger mounted in the airframe. It is important to note that the RS400 is not a scanning laser rangefinder. Scanning laser range finders are currently too heavy and consume too much power for MAV applications. The RS400 returns a single distance measurement and must be steered by maneuvering the airframe.

\section{Path Planning and Following}

The first step in our approach for navigating through complex environments is to plan a nominal path based on known information about the environment, which is usually in the form of a street map or topographic map. The MAV must be able to accurately follow the nominal path to avoid known obstacles. This section discusses the methods for planning and following the nominal path. Subsequent sections will discuss reactive, sensor-based obstacle avoidance strategies for obstacles unknown during the planning process.

\section{A. Planning the Nominal Path}

When planning paths through complex environments, the computational requirements for finding an optimal path can be significant and unrealistic for near-real-time execution [7]. Because of this, recent research has focused on randomized techniques to quickly find acceptable, though not necessarily optimal, paths [8], [9]. Path planning for MAVs is also difficult because of the dynamic constraints of flight. Many common path planning algorithms are inadequate for fixed-wing MAV systems because they do not handle turn-radius limitations and airspeed constraints effectively.

One randomized method that addresses these limitations is the Rapidly-exploring Random Tree (RRT) algorithm [7], [10]. RRTs use a dynamic model of the system to build a tree of traversable paths. The search space is quickly explored by applying control inputs to states already in the tree. Working with the precise control inputs ensures that the dynamic constraints are not violated; however, it also results in an open-loop solution. This would be adequate if we had a perfect model of the system and no disturbances, but this method is not satisfactory for an actual MAV because of model inaccuracies and disturbances, such as wind.

Similar to Frazzoli, et al. [11], we have extended some of the concepts of RRTs to plan paths in the output space. Through this work, we have developed a useful a priori path planner for the MAVs [12]. Our modified RRT algorithm searches the output states instead of the inputs and produces a list of waypoints to track. This is sufficient if we can bound the error of the controlled MAV from the waypoint path. For a given waypoint path, we can determine the expected trajectory of the MAV [13] and ensure that only traversable paths are built into the search tree. Branches in the tree are checked to ensure that they pass tests on turn radius and climb rate, and are collisionfree. Figure 5 depicts the growth of an RRT path through a simulated urban environment. A planned path through an actual canyon is shown in Figure 15.

\section{B. Vector Field Path Following}

Given a nominal waypoint path, it is essential for the MAV to have the ability to track the path with precision. MAVs must track these paths despite dynamic limitations, imprecise 


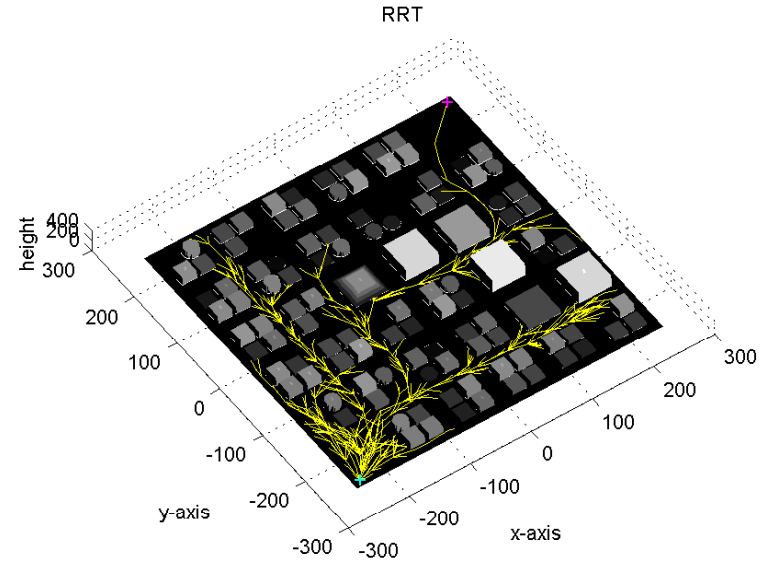

Fig. 5. This figure shows the growth of an RRT path tree through a simulated urban environment. The algorithm is terminated once a feasible path to the destination (red X) is found.

sensors and controls, and wind disturbances, which are often 20 to 60 percent of airspeed [14]. Trajectory tracking, which requires the MAV to be at a specific location at a specific time, is difficult in such wind conditions. As an alternative, we have developed a path following approach where the focus is simply to be on the path, instead of at a specific point that evolves in time. Similar research in [15] describes a maneuvering method focused on converging to the path then matching a desired speed along the path. Our path following method is based on the creation of course vector fields that direct the MAV onto the desired path.

The vector field method produces a field of desired course commands that drive the MAV toward the current path segment. At any point in space, the desired course can be easily calculated. This desired course is used to command heading and roll control loops to guide the MAV onto the desired path. The vector field method uses only the current path segment to find the desired course, avoiding possible singularities and sinks resulting from sums of vectors. Many paths planned for MAVs can be approximated by combinations of straight-line segments and circular arcs [16]. Figure 6 shows examples of vector fields for linear and circular paths.
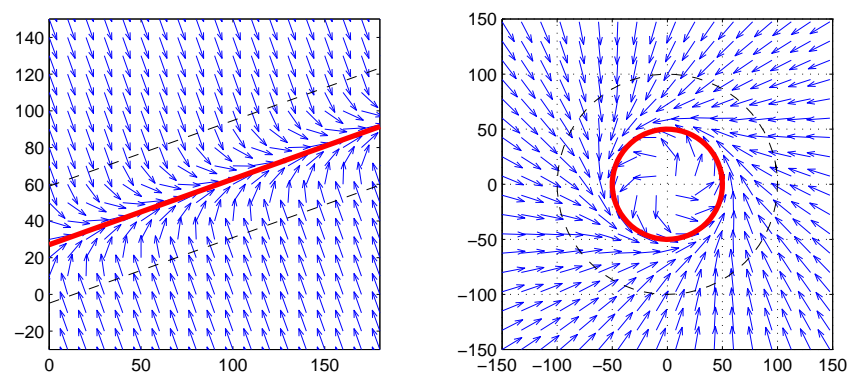

Fig. 6. Path following in wind is accomplished by creating a vector field of desired course commands based on the lateral deviation from the path. The figure on the left shows a possible vector field for a straight-line waypoint path segment. The figure on the right shows a possible vector field for orbit following.
To account for wind, we use the course and groundspeed instead of heading and airspeed to control the MAV. Groundtrack motion is the vector sum of the MAV motion relative to the surrounding air mass and the motion of the air mass relative to the ground. Since course direction includes the effects of wind, control based on course is much more effective at rejecting wind disturbances. In implementing the vector field approach, course measurements from GPS are compared with the desired course from the vector field to determine the appropriate control inputs to keep the MAV on the path.

For a given path, the vector field is divided into a transition region and an outer region. This is similar in some respects to the belt zone technique developed by Loizou, et al. [17] Outside the transition region, the vector field drives the MAV toward the transition region along a constant course. Once inside, the vector field changes linearly from the entry course direction to the desired course along the path. The effect is to smoothly drive the MAV to follow the path, with larger effort as the error from the path increases. In [14] it is shown that for any initial condition, the MAV will enter the transition region in finite time, then converge to the desired course asymptotically.

Flight tests have demonstrated the effectiveness of the vector field path following method, even in windy conditions. Figure 7 demonstrates path following for straight line segments with acute angles. Wind speeds were approximately 20 percent of the airspeed during these tests. The vector field method has been shown to be effective in tracking paths of lines and orbits with wind speeds of up to 50 percent of the airspeed of the MAV.

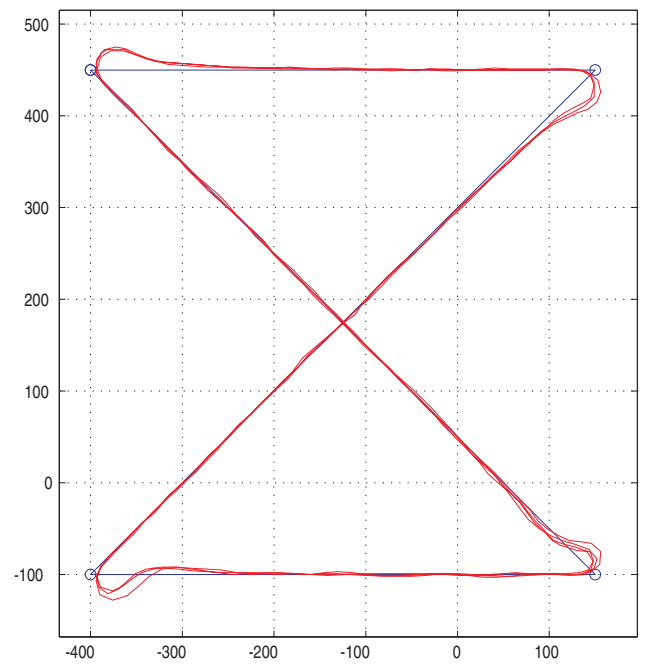

Fig. 7. This figure shows telemetry data for four consecutive traversals of a waypoint path. Wind speeds during the flight were $20 \%$ of the MAV airspeed. Note the repeatability of the trajectories even in significant wind.

\section{Reactive Obstacle and Terrain Avoidance}

Despite having an effective a priori path planner, we cannot guarantee that the flight path will be free of obstacles. Our path planner assumes a perfect model of the terrain, but this assumption is not realistic. If an urban terrain model is missing 
a newly constructed building or a large antenna or tree, a path leading to a collision could result. Our canyon models are based on 10 m USGS data, which is fairly accurate, but which cannot represent small obstacles like trees and power lines. In addition, the GPS sensor used on the MAV has a constant bias that can be as large as $10 \mathrm{~m}$. Path planners can produce a nominal path prior to flight, but the MAV must also have the ability to sense and reactively avoid unanticipated obstacles and terrain in real time.

The following sections present reactive planners for producing deviations from a nominal path to enable obstacle and terrain avoidance. Section IV presents a method for sensing and avoiding obstacles directly in the flight path and shows results for reactive avoidance of a building. Section $\mathrm{V}$ presents an approach for staying centered between obstacles as might be required for flying through a corridor. Flight test results are presented that demonstrate autonomous navigation of a winding canyon.

\section{Reactive Obstacle Avoidance}

Reactive obstacle avoidance from a MAV platform is challenging because of the size and weight limitations for sensing and computation hardware imposed by the platform. The speed with which avoidance decisions must be made and carried out also causes difficulties. For obstacle avoidance in urban environments, we have developed a heuristic algorithm that utilizes a laser ranger to detect and avoid obstacles. The laser ranger points directly out the front of the MAV, and returns range data for objects directly in front of the MAV with a $3 \mathrm{~Hz}$ update. For our preliminary flight tests, we considered a simple scenario: a single unknown obstacle placed directly in the flight path.

\section{A. Algorithm}

Consider the scenario shown in Figure 8 where obstacle avoidance is required. The MAV has a forward ground velocity $V$ and a minimum turn radius $R$ and is assumed to be tracking the given waypoint path at the time the obstacle is detected by the laser, which has a look ahead distance $L$. Figure 8 (a) shows the instant when the obstacle is detected by the laser ranger. The basic idea is to construct an internal map of obstacles detected by the laser and to modify the waypoint path to maneuver around the obstacles in the internal map. We will refer to the internal representation of obstacles as "map obstacles." When the laser detects the location of an obstacle, we are unsure about the size and height of the obstacle. We propose representing map obstacles as cylinders with radius $R$ equal to the minimum turn radius of the MAV, and height equal to the current altitude of the MAV. As shown in Figure 8 (b), there are two alternate waypoint paths that maneuver around the map obstacle. The endpoints of the waypoint paths are selected so that the new waypoint paths are tangent to the obstacles in the internal map. As shown in Figure 9 (a), the new waypoints are located a distance $d R / \sqrt{d^{2}-R^{2}}$ from the original waypoint path, where $d$ is the turn away distance from the obstacle. If both waypoint waypoint paths are collision free, then the algorithm randomly selects between the two paths as shown in Figure 8 (c). Since the map obstacle may be smaller than the the actual obstacle, the laser may again detect the obstacle as it maneuvers on the modified path. If that is the case, a new map obstacle is added to the internal map as shown in Figure 8 (d). This process is repeated until the MAV maneuvers around the obstacle as shown in Figures 8 (e) and (f).

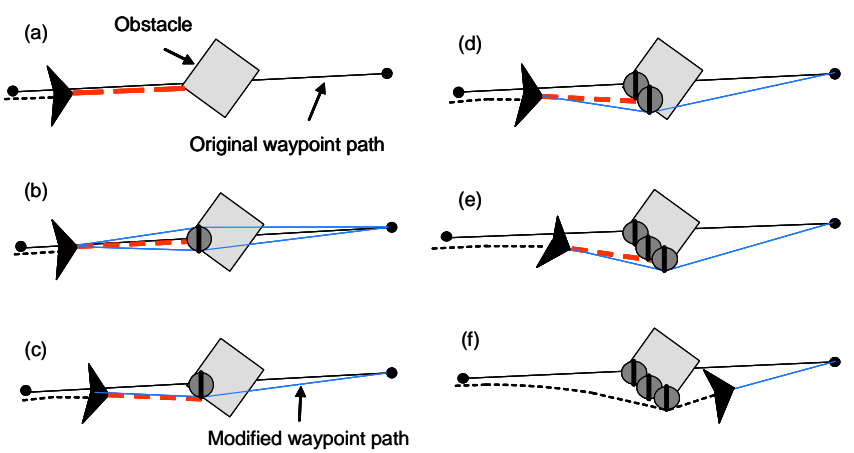

Fig. 8. Obstacle avoidance algorithm. (a) The laser detects the obstacle. (b) A map obstacle of radius $R$ is inserted into the map, and two candidate waypoint paths are constructed. (c) A modified waypoint path is randomly selected. (d) The obstacle is again detected by the laser and another map obstacle is constructed. (e-f) The process repeats until the MAV is able to maneuver around the obstacle.

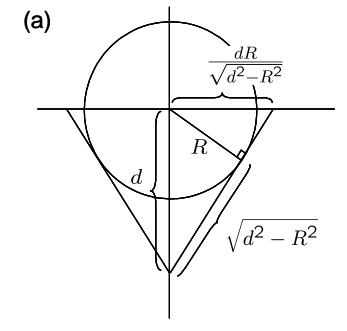

(b)

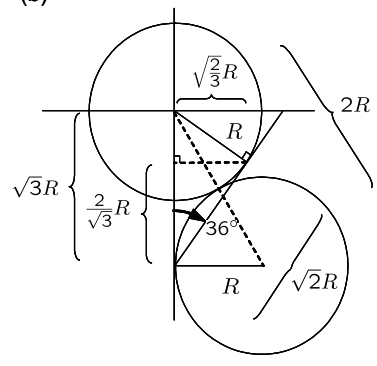

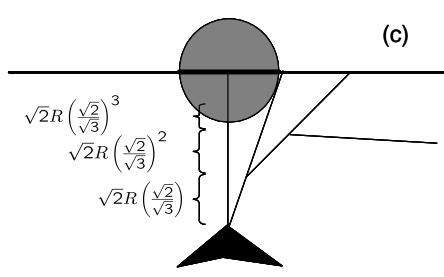

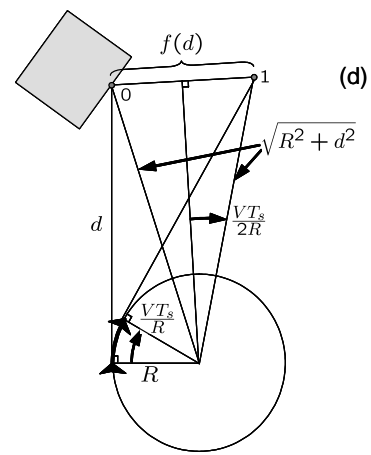

Fig. 9. (a) The waypoint path is constructed so that it is perpendicular to the map obstacle. The radius $R$ ensures collision free passage around the map obstacle. (b) The maximum heading change in waypoint paths is when the MAV must make a full bank to maneuver around the obstacle. (c) An approximation of the minimum distance required to avoid a straight wall if the laser is only sampled when the MAV is on the waypoint path. (d) the geometry used to calculate the distance between two consecutive laser updates.

If we assume zero wind, then the 2-D navigation for the MAV is given by

$$
\begin{aligned}
\dot{n} & =V \cos \chi \\
\dot{e} & =V \sin \chi \\
\dot{\chi} & =\frac{g}{V} \tan \phi,
\end{aligned}
$$


where $g$ is the gravitational constant, and $\phi$ is the roll angle of the MAV. On most MAVs, the roll angle is limited between $-\bar{\phi} \leq \phi \leq \bar{\phi}$. We will assume that the roll dynamics of the MAV are sufficiently fast to assume near instantaneous transitions between $\pm \bar{\phi}$. Therefore, the minimum turn radius is given by $R=\frac{V^{2}}{g \tan \phi}$.

We would like to establish a minimum turn away distance $D$ so that we are guaranteed to avoid collision with a single rectangular obstacle. The first step is to determine the bounds on the forward and lateral motion of the MAV when it transitions from one waypoint path to the next.

Claim: After the insertion of a map obstacle, the MAV requires at most a forward distance of $\frac{2}{\sqrt{3}} R$ and a lateral distance of $\sqrt{\frac{2}{3}} R$ to transition onto the new waypoint path while avoiding the map obstacle.

Assuming the ability to roll instantaneously between $\pm \bar{\phi}$, the motion of the MAV during the transition can be constrained to lie on circles of radius $R$. As shown in [13], the path length of the transition increases monotonically with the angle between the old and new waypoint paths. Therefore, the forward and lateral distances are maximized when the angular separation is maximized, which occurs when instantaneous motion of the MAV follows a circle of radius $R$ that just touches the map obstacle, as shown in Figure 9 (b). The claim follows directly from standard geometrical arguments. Note that the maximum angular separation is therefore given by $\theta=\tan ^{-1} \frac{1}{\sqrt{2}} \approx 36^{\circ}$.

Claim: Avoidance of a collision with a flat wall is guaranteed if the the turn away distance $D$ satisfies

$$
D>\left(\frac{8+2 \sqrt{6}}{2 \sqrt{3}}\right) R .
$$

Consider the worst-case scenario, shown in Figure 9 (c), of a MAV that is initially traveling perpendicular to a flat wall. The MAV detects an obstacle and inserts a waypoint at maximum angle $\tan ^{-1} \frac{1}{\sqrt{2}}$. After aligning its heading with the waypoint path, the wall is again detected, a map obstacle is inserted, and a new waypoint with maximum angle $\tan ^{-1} \frac{1}{\sqrt{2}}$ is planned. This scenario will repeat itself at most three times since $3 \tan ^{-1} \frac{1}{\sqrt{2}}>\frac{\pi}{2}$. Therefore, the maximum forward direction is bounded by

$$
\sqrt{2} R\left(\left(\sqrt{\frac{2}{3}}\right)^{1}+\left(\sqrt{\frac{2}{3}}\right)^{2}+\left(\sqrt{\frac{2}{3}}\right)^{3}\right)=\left(\frac{8+2 \sqrt{6}}{2 \sqrt{3}}\right) R .
$$

We note that the algorithm described above, requires that the laser detect points on the obstacle that are outside of the map obstacles as soon as they become visible. Is this feasible given the update rate of the laser? Let $T_{s}$ be the time between laser updates.

Claim: The maximum distance between laser updates at a range of $d \leq L$ is given by

$$
f(d)=2 \sqrt{R^{2}+d^{2}} \sin \left(\frac{V T_{s}}{2 R}\right)
$$

Assuming the vehicle is turning at its maximum rate, the change in heading between updates is $\frac{V T_{s}}{R}$. Utilizing the geometry depicted in Figure 9 (d), the calculation of $f(d)$ is straightforward. To ensure overlap of map obstacles between samples we require that $f(D)<R$ which implies that

$$
T_{s}<\frac{2 R}{V} \sin ^{-1}\left(\frac{R}{2 \sqrt{R^{2}+D^{2}}}\right) .
$$

For our airframes, typical values are $V=13 \mathrm{~m} / \mathrm{s}, R=$ $25 \mathrm{~m}$, which implies from (1) that $D=93 \mathrm{~m}$ and $T_{s}<$ $0.5 \mathrm{~s}$. The laser ranger sample period of $0.33 \mathrm{~s}$ satisfies this constraint, thus ensuring that map obstacles overlap between samples.

\section{B. Results}

For initial testing of the reactive avoidance algorithm, we chose to deal with a single obstacle only. It was important that the obstacle be tall enough to allow the MAV to fly at a safe altitude. Flying at an altitude of $40 \mathrm{~m}$ also prevented the laser ranger from detecting points on the ground that might be mistakenly interpreted as obstacles, and allowed for losses of altitude that can occur during aggressive maneuvers.

For our flight tests, we used the tallest building on the BYU campus (the Kimball Tower) which is $50 \mathrm{~m}$ high and $35 \mathrm{~m}$ square and is shown in Figure 11. The surrounding buildings are only about $20 \mathrm{~m}$ in height. The MAV was directed to fly at $40 \mathrm{~m}$ altitude from the south side of the building to the north along a waypoint path that passed directly through the building. No information about the location or the dimensions of the building were provided to the MAV. A GPS telemetry plot of the results is shown in Figure 10.

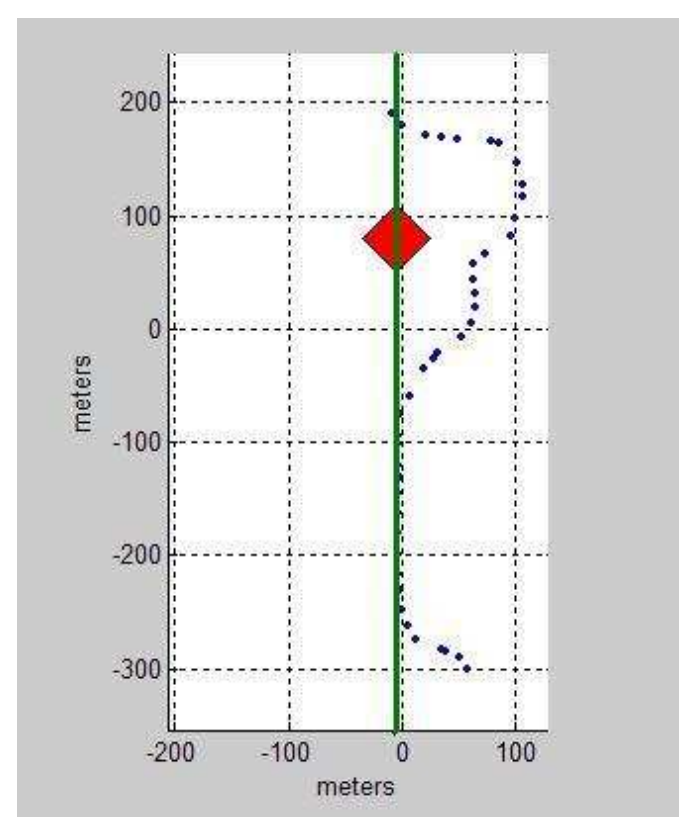

Fig. 10. Flight results for collision avoidance using a laser ranger. The green line indicates the planned waypoint path, and the dotted line indicates the GPS track of the MAV.

As the MAV approached the building, the laser ranger detected the building and calculated its position. When the MAV came within $93 \mathrm{~m}$ of the building, the reactive planner generated a path around the building and the MAV began to 
track the path. Notice that as the MAV began to pass the building, it turned towards the original waypoint path and detected the building a second time. This caused the MAV to execute a second avoidance maneuver before rejoining the original waypoint path. The MAV successfully avoided the building without human intervention. Figure 11 shows images of the MAV and its camera view as it executed the avoidance maneuver.

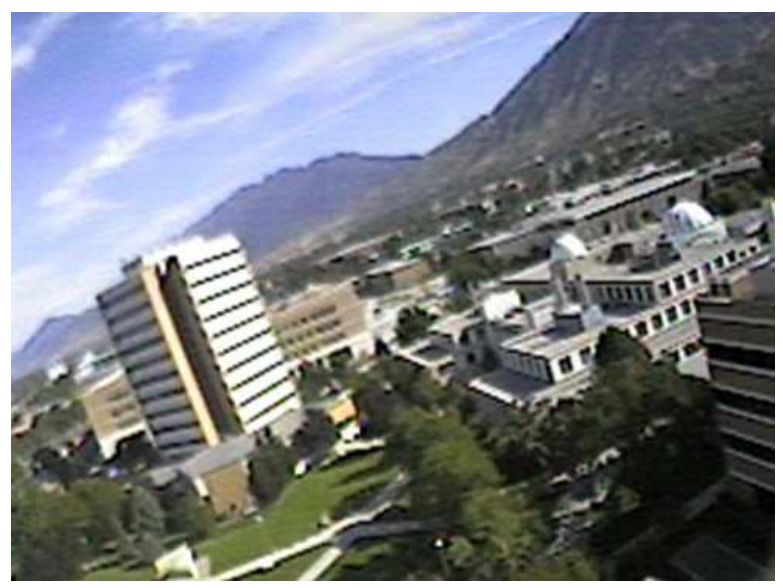

Fig. 11. In-flight image of the Kimball Tower on BYU campus during the collision avoidance maneuver.

\section{Remote EnVironment Terrain AVoidance}

As small MAVs become more reliable and maneuverable, their missions will involve navigating through complex terrain, such as mountainous canyons and urban environments. In this section, we focus on terrain avoidance for flying in corridors and canyons. The algorithms we have developed enable the MAV to center itself within a corridor or canyon, or to fly near walls with a specified offset. The algorithms utilize optic flow sensors like those shown in Figure 3. To validate our algorithms, canyon navigation flight experiments were carried out in a mountain canyon.

\section{A. Canyon Navigation Algorithm}

The first step in navigating through a canyon or urban corridor is to select a suitable path through the terrain. This can be done using the RRT algorithm discussed earlier or the operator can utilize maps to define waypoints for the MAV to follow. Preplanned paths will rarely be perfect and some paths could lead the MAV near or even into uncharted obstacles. Reasons for this include inaccurate or biased terrain data, GPS error, and the existence of obstacles that have been added since the terrain was mapped. Therefore, it is important that the MAV be able to make adjustments to its path to center itself between walls and other potential hazards.

In our approach, the MAV follows its preplanned path using the vector field following method. At each time step along the path the MAV computes its lateral distance from objects to the left and right using the optic flow ranging sensors. Using this information, the MAV computes an offset $\delta$ from its planned path

$$
\delta=\frac{1}{2}\left(D_{\text {right }}-D_{\text {left }}\right),
$$

where $D_{\text {left }}$ and $D_{\text {right }}$ are distances to walls on the left and right measured by the optic flow sensors. Shifting the desired path by this offset centers the desired path between the detected walls as shown in Figure 12. As Figure 13 illustrates, shifting the desired path also shifts the vector field accordingly. To improve the performance of this method the optic ranging sensors are pointed forward at a 30 degree angle. This reduces lag caused by filtering the sensor readings and allows the MAV to detect obstacles ahead of its current position.

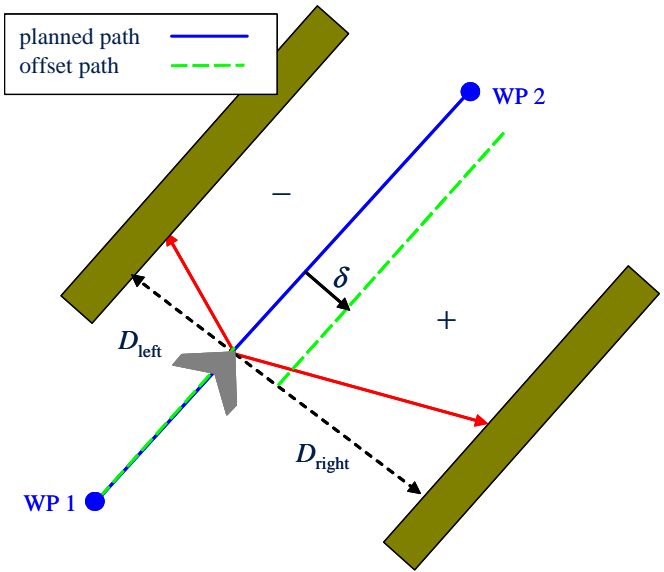

Fig. 12. Using the measurements from the optic flow sensors, the planned path (solid blue) is shifted by $\delta$ to create a new desired path (dashed green) that is centered between the canyon walls.

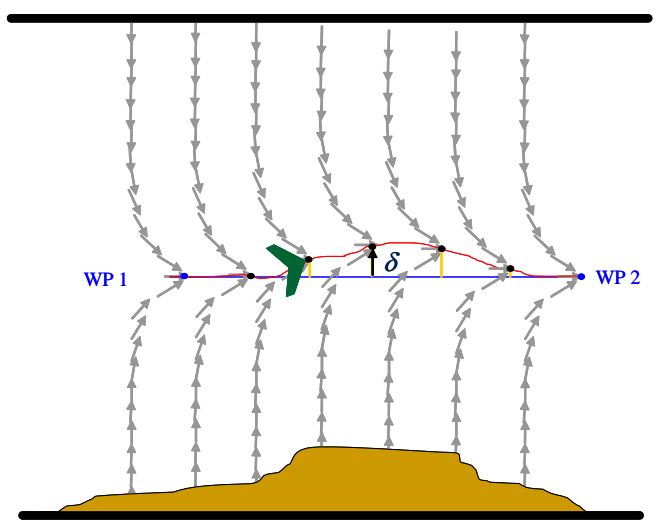

Fig. 13. The adjusted path (red) is offset from the preplanned path (blue) by the calculated offset $(\delta)$ at each time step to center the desired path between the canyon walls, thus shifting the vector field along with it.

\section{B. Flight Test Results}

Goshen Canyon in central Utah was chosen as a flight test site. This canyon was selected for its steep winding canyon walls that reach over $75 \mathrm{~m}$ in height, as well as its proximity to BYU and low utilization. Flight tests through Goshen Canyon were conducted using the fixed-wing MAV 
discussed in Section II. Photographs of the flight tests taken by observers and the onboard camera are shown in Figure 14. In the first flight through the canyon, the planned path was selected to follow the road. The MAV navigated the canyon with only minor adjustments to its path. For the second flight, the planned path was intentionally biased into the east canyon wall to verify that the navigation algorithms would correct the planned path toward the center of the canyon, enabling the MAV to avoid the canyon walls.

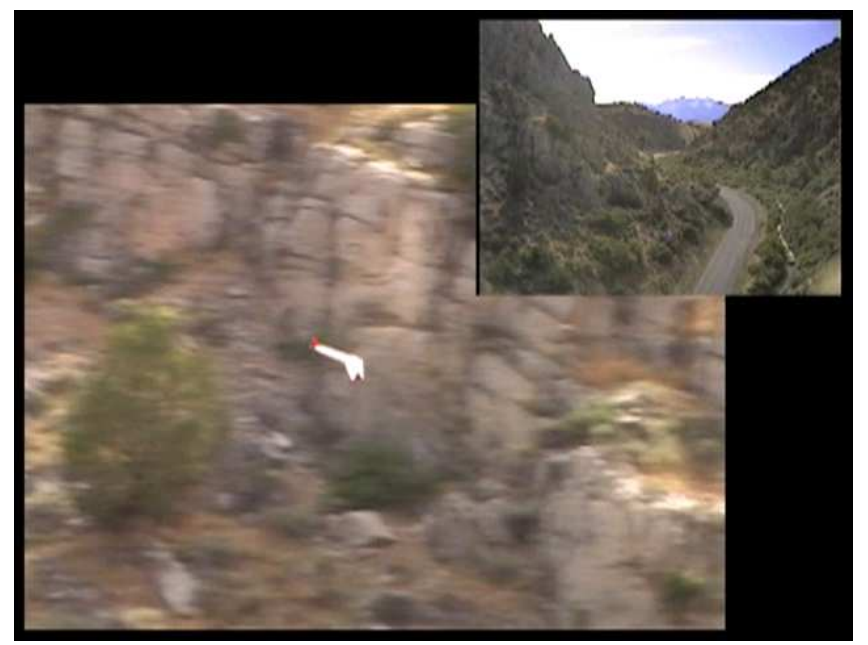

Fig. 14. This figure shows the MAV as it enters Goshen Canyon. The inset is an image from the camera on-board the MAV.

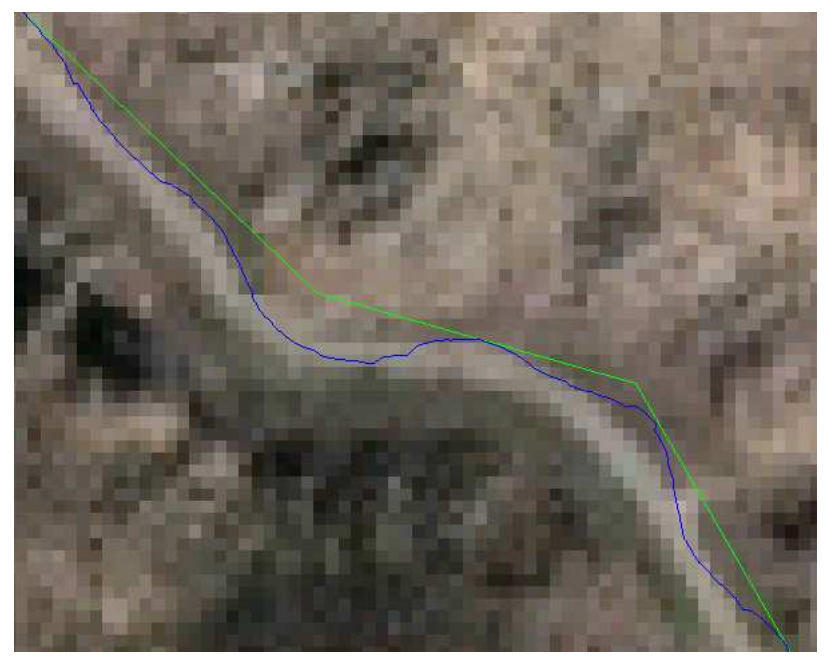

Fig. 15. Results from the second flight through Goshen Canyon. Flight test results show the planned path (green) and the actual path (blue). The planned path was intentionally biased to the east forcing the MAV to offset from its planned path to center itself through the canyon.

Figures 15 shows results from the second flight which demonstrate that the MAV biased its desired path up to $10 \mathrm{~m}$ to the right to avoid the canyon walls. If the MAV had not biased its path it would have crashed into the east canyon wall.

\section{SUMMARY}

Miniature aerial vehicles have demonstrated their potential in numerous applications. Even so, they are currently limited to operations in open air space, far away from obstacles and terrain. To broaden the range of applications for MAVs, methods to enable operation in environments of increased complexity must be developed. In this article, we presented two strategies for obstacle and terrain avoidance that provide a means for avoiding obstacles in the flight path and for staying centered in a winding corridor. Flight tests have validated the feasibility of these approaches and demonstrated promise for further refinement.

\section{ACKNOWLEDGMENT}

This work was funded AFOSR award numbers FA9550-041-0209 and FA9550-04-C-0032.

\section{REFERENCES}

[1] J. Chahl, M. Srinivasan, and S. Zhang, "Landing strategies in honeybees and applications to uninhabited airborne vehicles," The International Journal of Robotics Research, vol. 23, no. 2, pp. 101-110, 2004.

[2] G. Barrows and C. Neely, "Mixed-mode VLSI optic flow sensors for inflight control of a micro air vehicle," in Proceedings SPIE, San Diego, August 2000, pp. 52-63.

[3] F. Ruffier and N. Franceschini, "Visually guided micro-aerial vehicle: automatic take off, terrain following, landing and wind reaction," in Proceedings of the 2004 IEEE International Conference on Robotics \& Automation, New Orleans, 2004, pp. 2339-2346.

[4] J.-C. Zufferey and D. Floreano, "Toward 30-gram autonomous indoor aircraft: Vision-based obstacle avoidance and altitude control," in Proceedings of the 2005 IEEE International Conference on Robotics \& Automation, Barcelona, April 2005.

[5] G. L. Barrows, J. S. Chahl, and M. V. Srinivasan, "Biomimetic visual sensing and flight control," The Aeronautical Journal, London: The Royal Aeronautical Society, vol. 107, pp. 159-168, 2003.

[6] Procerus Technologies, http://procerusuav.com/.

[7] S. M. LaValle and J. J. Kuffner, "Randomized kinodynamic planning," International Journal of Robotic Research, vol. 20, no. 5, pp. 378-400, May 2001.

[8] L. E. Kavraki, P. Švestka, J.-C. Latombe, and M. Overmars, "Probabilistic roadmaps for path planning in high-dimensional configuration spaces," IEEE Transactions on Robotics and Automation, vol. 12, no. 4, pp. 566-580, 1996.

[9] N. M. Amato and Y. Wu, "A randomized roadmap method for path and manipulation planning," in Proceedings of the IEEE International Conference on Robotics and Automation, Minneapolis, MN, 1996, pp. 113-120.

[10] S. M. LaValle, "Rapidly-exploring random trees: A new tool for path planning," October 1998, tR 98-11, Computer Science Dept., Iowa State University.

[11] E. Frazzoli, M. A. Dahleh, and E. Feron, "Real-time motion planning for agile autonomous vehicles," AIAA Journal of Guidance, Control and Dynamics, vol. 25, no. 1, pp. 116-129, 2002.

[12] J. B. Saunders, B. Call, A. Curtis, R. W. Beard, and T. W. McLain, "Static and dynamic obstacle avoidance in miniature air vehicles," in Proceedings of the Infotech@Aerospace Conference, September 2005.

[13] E. P. Anderson, "Constrained extremal trajectories and unmanned air vehicle trajectory generation," Master's thesis, Brigham Young University, Provo, Utah, April 2002.

[14] D. R. Nelson, "Cooperative control of miniature air vehicles," Master's thesis, Brigham Young University, Provo, Utah, December 2005.

[15] R. Skjetne, T. Fossen, and P. Kokotović, "Robust output maneuvering for a class of nonlinear systems," Automatica, vol. 40, pp. 373-383, 2004.

[16] E. P. Anderson, R. W. Beard, and T. W. McLain, "Real time dynamic trajectory smoothing for uninhabited aerial vehicles," IEEE Transactions on Control Systems Technology, vol. 13, no. 3, pp. 471-477, May 2005.

[17] S. Loizou, H. Tanner, V. Kumar, and K. Kyriakopoulos, "Closed loop motion planning and control for mobile robots in uncertain environments," in Proceedings of the 42nd IEEE Conference on Decision and Control, 2003, pp. 2926-2931. 